\title{
Speed and Direction Angle Control of Four Wheel Drive Skid-Steered Mobile Robot by Using Fractional Order PI Controller
}

\author{
Kamil Orman ${ }^{1}$, Abdullah Basci ${ }^{2}$, Adnan Derdiyok ${ }^{3}$ \\ ${ }^{l}$ Department of Electronics and Automation, Erzincan University, Vocational High School, \\ Erzincan \\ ${ }^{2}$ Faculty of Engineering, Atatürk University, Electrical and Electronics Engineering, \\ Erzurum \\ ${ }^{3}$ Faculty of Technology, Sakarya University, Electrical and Electronics Engineering, \\ Sakarya \\ abasci@atauni.edu.tr
}

\begin{abstract}
${ }^{1}$ Abstract-In this paper, speed and direction angle control of four-wheel drive skid-steered mobile robot (4WD SSMR) is realized by Fractional-Order Proportional Integral (FOPI) controller. Speed and direction angle of the mobile robot are calculated by using angular velocity of each motors. FOPI controller produces the torques of each motor of mobile robot for trajectory tracking and stabilization in the desired position. A well-tuned conventional PI controller is also applied to mobile robot for comparison with the FOPI. Experimental results prove that the FOPI shows better trajectory tracking performance than PI controller in terms of trajectory tracking accuracy and error levels.
\end{abstract}

Index Terms-4WD SSMR; fractional order PI; BLDC motor; trajectory tracking.

\section{INTRODUCTION}

In the past few years, researchers have focused on the automated guided vehicle (AGV) trajectory tracking problems and different approaches have been discussed. Especially, the nonholonomic constraints of AGV have been taken into consideration. Normey-Rico et al. [1] have proposed a path tracking controller based on a robust PID algorithm. Their method uses a simple linearized model of the mobile robot composed of an integrator and a delay. They used an easy synthesis procedure and the obtained rules were similar to the Ziegler-Nichols method for PID controllers. Zhao et al. [2] were developed a suitable reference model based on the vehicle parameters for Autonomous vehicle by simplified bicycle model of an automobile. In addition, they used the adaptive PID control system for adaptability and stability. Tamogna and Kar [3] have proposed a control structure that makes possible the integration of a kinematic controller and an adaptive fuzzy controller for trajectory tracking control of nonholonomic mobile robots. In their method, adaptive controller used a Fuzzy Logic System for estimating the nonlinear robot functions involving unknown robot parameters for tracking

Manuscript received 29 April, 2016; accepted 15 August, 2016. control of wheeled mobile robots. Huang et al. [4] suggested a high-gain observer based adaptive output feedback tracking control design scheme for nonholonomic mobile robots. They used observers to estimate the unknown linear and angular velocities respectively. Peng et al. [5] have discussed in the mobile device two subsystem, that is, nonholonomic mobile platform and holonomic manipulator subsystem. They derived a kinematic controller for two subsystem to obtain a desired velocity by Lyapunov functions. Also, they proposed a robust adaptive tracking controller and according to the Lyapunov stability theory, the tracking errors and adaptive coefficient errors are all bounded.

Although fractional order calculation has an old history besides all these developments, today it can be considered a new issue. Its onset is based on G.W. Leibnz (1695) and L. Euler (1730). It is presented basic math of fractional calculus [6], solutions of fractional order differential equations [7], [8] some provisions in the engineering of these systems [9]. Thus FOPI controllers are increasingly becoming popular and have been gained many working area especially in control applications [10]-[14]. Studies have shown that the fractional order systems can produce much better results than integer order system. The FOPI control is robust in systems including uncertainty. Therefore, it is necessary to determine the optimum parameters. For solution, it is presented the electromagnetism and evolutionary optimization algorithms [15], fractional-order controller design using genetic algorithms [16], particle swarm optimization algorithm [17], an iterative optimization method according to nonlinear function minimization [18], an auto-tuning method for the fractional order $\mathrm{PI}^{\lambda} \mathrm{D}^{\mu}$ controller using the relay test [19]. These proposed methods allow direct selection of the parameters of the controller through the knowledge of the magnitude and phase of the plant at the frequency of interest obtained with the relay test and a practical and systematic tuning procedure [20].

In this paper, a FOPI controller and a well-tuned conventional PI controller are applied to experimental setup 
of four-wheel skid-steering mobile robot for speed and direction angle control, respectively. The determination of the FOPI and PI controller parameters are achieved by trial and error. The experimental results showed that the FOPI has given better results than the PI controller in terms of trajectory tracking accuracy and error levels.

\section{4WD SSMR DESIGN}

Four-wheel skid-steering mobile robot (moving on two dimensional plane with inertial coordinate frame) is depicted in Fig. 1. The kinematic equations of the skid-steered mobile robot are derived as follows [21]-[23].

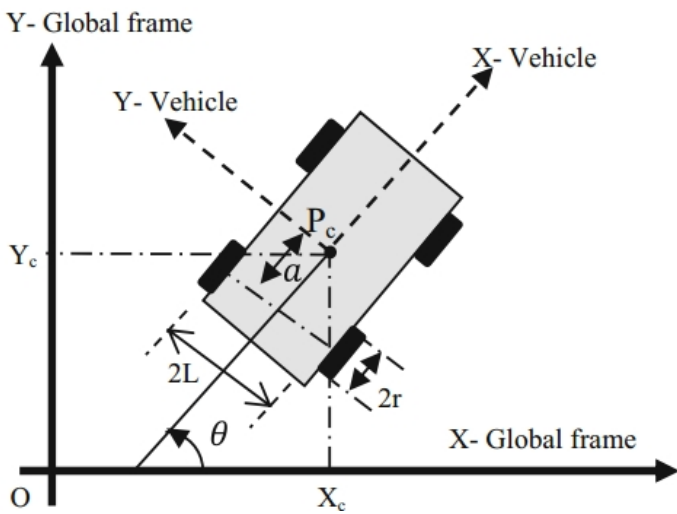

Fig. 1. Kinematics of 4 WD SSMR.

The vehicle has four fixed wheels and wheels are skidsteer motion and each wheel is driven by a Brushless DC motor through a gear box as shown in Fig. 2.

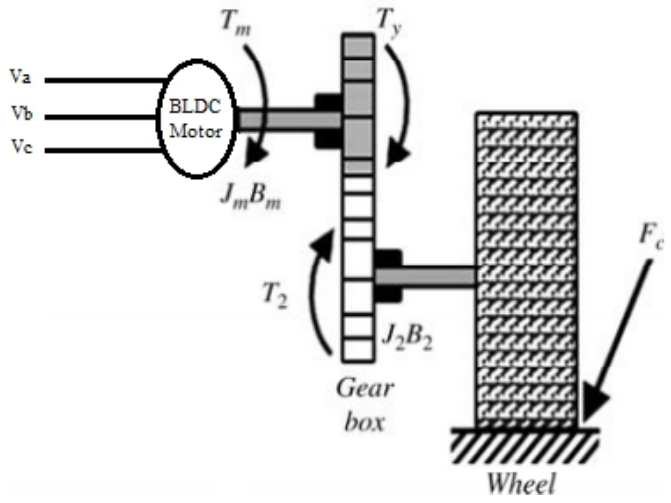

Fig. 2. Drive system of each wheel.

$$
\begin{gathered}
{\left[\begin{array}{c}
\dot{x} \\
\dot{y} \\
\dot{\theta}
\end{array}\right]=\left[\begin{array}{cc}
\frac{r}{2} & \frac{r}{2} \\
0 & 0 \\
\frac{r}{L} & -\frac{r}{L}
\end{array}\right]\left[\begin{array}{c}
\omega_{r} \\
\omega_{l}
\end{array}\right],} \\
v=r \times\left[\frac{\omega_{r}+\omega_{l}}{2}\right], \\
\dot{\theta}=\omega=r \times\left[\frac{\omega_{r}+\omega_{l}}{L}\right], \\
\left\{\begin{array}{l}
\dot{\theta}_{r}=\omega_{r}, \\
\dot{\theta}_{l}=\omega_{l}, \\
v_{x}=v \times \cos (\theta)=\mathrm{r} \times\left[\frac{\omega_{r}+\omega_{l}}{2}\right] \times \cos (\theta),
\end{array}\right.
\end{gathered}
$$

$$
v_{y}=v \times \sin (\theta)=\mathrm{r} \times\left[\frac{\omega_{r}+\omega_{l}}{2}\right] \times \sin (\theta) .
$$

No slip constraint

$$
\dot{y}_{c} \times \cos (\theta)-\dot{x}_{c} \times \sin (\theta)=\alpha \dot{\theta} .
$$

Pure rolling constraint:

$$
\begin{aligned}
& \dot{x}_{c} \times \cos (\theta)+\dot{y}_{c} \times \sin (\theta)+L \dot{\theta}=r \dot{\theta}_{r}, \\
& \dot{x}_{c} \times \cos (\theta)+\dot{y}_{c} \times \sin (\theta)-L \dot{\theta}=r \dot{\theta}_{l},
\end{aligned}
$$

where ' $\mathrm{r}$ ' is radius of wheels, ' $2 \mathrm{~L}$ ' is the distance between the left and right wheels, $\omega_{r}$ and $\omega_{l}$ are right and left wheels' angular velocity, $v$ and $\theta$ are speed and direction angle of 4WD SSMR, $v_{x}$ and $v_{y}$ are speed component of centre of gravity of the $4 \mathrm{WD} \operatorname{SSMR}\left(\mathrm{P}_{\mathrm{c}}\right)$ for $x$ and $y$ directions. The actual position of the 4WD SSMR is represented by generalized coordinates, $P_{c}=\left(x_{c}, y_{c}, \theta\right)$.

\section{A. Obtaining the Transfer Function of BLDC Motors}

Firstly, consider the equation of the DC motor to obtain the transfer function of the BLDC motor. The voltage equation and transfer function of DC motor can be expressed as [24]

$$
V=L \frac{d i}{d t}+R i+E
$$

where:

$$
\begin{gathered}
E=K_{e} \frac{d \phi}{d t}, \\
L \frac{d i}{d t}=V-R i-K_{e} \frac{d \phi}{d t}, \\
i K_{t}=J_{m} \frac{d^{2} \phi}{d t^{2}}+B_{m} \frac{d \phi}{d t} .
\end{gathered}
$$

The transfer function is obtained using (12) and (13) in sform

$$
G(s)=\frac{K_{t}}{L J_{m} s^{2}+\left(J_{m} R+B_{m} L\right) s+R B_{m}+K_{t} K_{e}} .
$$

We can simplify (22) considering the following assumptions:

1. $B_{m}$ is small and in that case its tends to 0 , so,

2. $\left.\left.J_{m} R\right\rangle\right\rangle B_{m} L$,

3. $\left.K_{t} K_{e}\right\rangle>R B_{m}$.

If the negligible values are eliminated, (14) written as

$$
G(s)=\frac{K_{t}}{L J_{m} s^{2}+J_{m} R s+K_{t} K_{e}},
$$

by multiplying top and bottom of (15) by 


$$
\frac{R}{K_{t} K_{e}} \times \frac{1}{R}
$$

Transfer function

$$
G(s)=\frac{\frac{1}{K_{e}}}{\frac{R J_{m}}{K_{t} K_{e}} \frac{L}{R} s^{2}+\frac{R J_{m}}{K_{t} K_{e}} s+1}
$$

where ' $K_{t}$ ' is the torque constant $(m N m / A)$, ' $K_{e}$ ' is electrical torque (Vsec/rad), ' $\mathrm{L}$ ' is Terminal inductance phase to phase $(m H)$, 'Bm' is frictional coefficient of motor and load $(\mathrm{Nm} /(\mathrm{rad} / \mathrm{sec}))$, ' $\mathrm{Jm}$ ' is the motor inertia $\left(\mathrm{Kgm}^{2}\right)$, ' $R$ ' is Terminal resistance phase to phase $(\Omega)$. The mechanical time constant

$$
\tau_{m}=\frac{R J_{m}}{K_{t} K_{e}} .
$$

The electrical time constant

$$
\tau_{e}=\frac{L}{R}
$$

Substituting (18) and (19) into (17)

$$
G(s)=\frac{\frac{1}{K_{e}}}{\tau_{m} \tau_{e} s^{2}+\tau_{m} s+1},
$$

so (18) and (19) indicate the difference between DC and Brushless DC motors. The mechanical and electrical constants are very important parts of motor model parameters. Given the symmetrical structure, the mechanical time constant (18) becomes [25]

$$
\tau_{m}=\sum \frac{R J_{m}}{K_{t} K_{e}}=\frac{J_{m} \sum R}{K_{t} K_{e}} .
$$

The electrical time constant

$$
\tau_{e}=\sum \frac{L}{R}=\frac{L}{\sum R} .
$$

There is a symmetrical arrangement a three phases. Therefore, the mechanical and electrical constants become:

$$
\left\{\begin{array}{l}
\tau_{m}=\frac{J_{m} 3 R}{K_{t} K_{e}} \\
\tau_{e}=\frac{L}{3 R}
\end{array}\right.
$$

Also, using the electrical power and mechanical power equations; the relationship between $K_{e}$ and $K_{t}$ is expressed as follows:

$$
\begin{gathered}
\sqrt{3} \times E \times I=\frac{2 \pi}{60} \times N \times T, \\
\frac{E}{N}=\frac{T}{I} \times \frac{2 \pi}{60 \times \sqrt{3}}, \\
K_{e}=K_{t} \times \frac{2 \pi}{60 \sqrt{3}}=K_{t} \times 0.0605 .
\end{gathered}
$$

\section{B. Maxon Motor (EC 32), Brushless DC Motor}

TABLE I. SPECIFICATION OF MAXON BLDC MOTOR (118889).
\begin{tabular}{|c|c|c|}
\hline S. No. & Parameter(unit) & Specification \\
\hline 1 & Nominal voltage $(\mathrm{V})$ & 24 \\
\hline 2 & No load speed $(\mathrm{rpm})$ & 11000 \\
\hline 3 & No load current $(\mathrm{mA})$ & 286 \\
\hline 4 & Nominal speed $(\mathrm{rpm})$ & 9510 \\
\hline 5 & Nominal torque $(\mathrm{mNm})$ & 43.6 \\
\hline 6 & Nominal current $(\mathrm{A})$ & 3.37 \\
\hline 7 & Stall torque $(\mathrm{mNm})$ & 355 \\
\hline 8 & Starting current $(\mathrm{A})$ & 17.3 \\
\hline 9 & Maximum efficiency & $76 \%$ \\
\hline
\end{tabular}

TABLE II.CHARACTERISTICS OF MAXON BLDC MOTOR (118889).

\begin{tabular}{|c|c|c|}
\hline S. No. & Parameter(unit) & Specification \\
\hline 1 & $\begin{array}{c}\text { Terminal resistance phase to phase } \\
(\Omega)\end{array}$ & 1.39 \\
\hline 2 & $\begin{array}{c}\text { Terminal inductance phase to } \\
\text { phase }(\mathrm{mH})\end{array}$ & 0.226 \\
\hline 3 & Torque constant $(\mathrm{mNm} / \mathrm{A})$ & 20.5 \\
\hline 4 & Speed constant $(\mathrm{rpm} / \mathrm{V})$ & 465 \\
\hline 5 & Speed/torque gradient $(\mathrm{rpm} / \mathrm{mNm})$ & 31.5 \\
\hline 6 & Mechanical time constant $(\mathrm{ms})$ & 6.59 \\
\hline 7 & Rotor inertia $\left(\mathrm{gcm}^{2}\right)$ & 20 \\
\hline
\end{tabular}

The mathematical model of the BLDC motor is modelled based on the parameters from table 1-2, the values for $K_{e}$, $\tau_{m}$ and $\tau_{e}$ need to calculated:

$$
\begin{gathered}
-R=1.39 \Omega ; \\
-J_{m}=20 \mathrm{gcm}^{2}=2 \times 10^{-6} \mathrm{Kgm}^{2} ; \\
-K_{t}=20,5 \mathrm{mNm} / \mathrm{A}=20.5 \times 10^{-3} \mathrm{Nm} / \mathrm{A} ; \\
-\tau_{m}=6.59 \mathrm{~ms}=0.00659 \mathrm{~s} . \\
\tau_{e}=\frac{L}{3 R}=\frac{0.226 \times 10^{-3}}{3 \times 1.39}=54.19 \times 10^{-6}, \\
\tau_{m}=\frac{J_{m} 3 R}{K_{t} K_{e}}=0.00659[\mathrm{~s}], \\
K_{e}=\frac{J_{m} 3 R}{K_{t} \tau_{m}}=\frac{2 \times 10^{-6} \times 3 \times 1.39}{20.5 \times 10^{-3} \times 0.00659}, \\
K_{e}=0.06173[\mathrm{~V} \mathrm{sec} / \mathrm{rad}] .
\end{gathered}
$$

Using (20) G(s) becomes:

$$
\begin{gathered}
G(s)=\frac{\frac{1}{0.06173}}{0.00659 \times 54.19 \times 10^{-6} \times s^{2}+0.00659 \times s+1}, \\
G(s)=\frac{16.19}{0.357 \times 10^{-6} \times s^{2}+0.00659 \times s+1} .
\end{gathered}
$$




\section{FRACTIONAL ORDER CONTROLLER}

The fractional-order differentiator can be denoted by a general fundamental operator ${ }_{a} D_{t}^{p}$, where $a$ and $t$ are the limits of operations. The fractional-order differentiator and integral are defined as follows

$$
{ }_{a} D_{t}^{p}=\left\{\begin{array}{c}
\frac{d^{p}}{d t^{r}}, \mathrm{p}>0 \\
1, \mathrm{p}=0 \\
t t(\mathrm{~d} \tau)^{-\mathrm{p}}, \mathrm{p}<0 \\
a a
\end{array}\right.
$$

where $p$ is the fractional order which can be a complex number, however the constant $p$ is related to initial conditions. There are several mathematical definitions to describe the fractional derivatives and integrals [6], [7]. Between these definitions, here are two commonly used ones, i.e., the Grünwald-Letnikov (GL) and the RiemannLiouville (RL). The GL definition is

$$
{ }_{a} D_{t}^{p} f(t)=\lim _{h \rightarrow 0} h^{-p^{\left[\frac{t-a}{h}\right.}} \sum_{j=0}(-1)^{j}\left(\begin{array}{l}
p \\
j
\end{array}\right) f(t-j h),
$$

where [.] means the integer part, while the RL definition is given as

$$
{ }_{a} D_{t}^{p} f(t)=\frac{1}{\Gamma(\mathrm{n}-\mathrm{p})} \frac{d^{n}}{d t^{n}} \int_{a}^{t} \frac{f(\tau)}{(t-\tau)^{p-n+1}} d \tau
$$

for $(\mathrm{n}-1<p<n), \Gamma($.$) is the Euler's gamma function, a$ is the initial time and $t$ parameter is used when the differential and integral are taken. The general form of the fractional order PI controller is the $P I^{\lambda}$ and its general transfer function is given as

$$
C(s)=K_{p}+\frac{K_{i}}{s^{\lambda}}
$$

where $\lambda$ is fractional order, $K_{p}$ and $K_{i}$ are the proportional gain and integration constant respectively. The optimization of the tree parameters $K_{p}, K_{i}$ and $\lambda$ makes designing of FOPI controller more challenging than integer order PI controller. Several methods are proposed for optimization. In this paper the determination of the tree parameters is achieved by trial and error.

\section{CONTROL OF 4WD SSMR}

The control method is explained as shown in Fig. 3. The required torque for each motor is computed by $u_{v}$ and $u_{\theta}$ that are defined as outputs of controller. Torque of the right front and back wheels

$$
\tau_{r}=\frac{u_{v}+u_{\theta}}{2}
$$

Torque of the left front and back wheels

$$
\tau_{l}=\frac{u_{v}-u_{\theta}}{2}
$$

4WD SSMR speed and direction angle is calculated from the equation (2) and (3) given for left and right motors. Considering (25) and (26), $u_{v}$ and $u_{\theta}$ signals are generated by the controller to calculate the right and left reference torque.

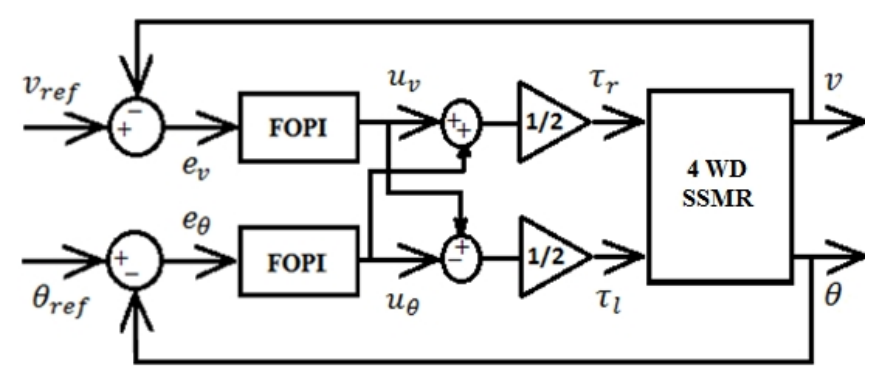

Fig. 3. Block diagram of the 4WD SSMR control system.

\section{EXPERIMENTAL RESULT}

In this section, the performance of the FOPI controller is achieved by comparing the speed and direction angle of 4WD SSMR with well-tuned PI controller results. The both controllers are executed on the 4WD SSMR shown in Fig. 4. The 4WD SSMR is equipped with four fast response Brushless DC motors with incremental encoders counting 500 pulses/turn and speed reductions gear boxes and an industrial PC with DAQ.

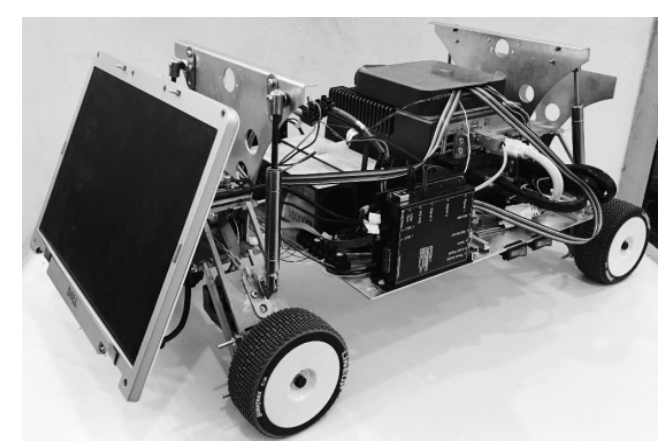

Fig. 4. 4WD SSMR platform: actual view.

In order to show performance of the proposed controller, three experiments have been conducted and results are shown in Fig. 5- Fig. 7.

In the first experiment, a sinusoidal speed and sinusoidal direction angle references are chosen for testing FOPI and PI controllers. As shown in Fig. 5, the both controller have similar responses and the maximum percentage errors of sine wave reference for FOPI is $4.1 \%$ and for PI is $5.9 \%$ respectively. Due to chosen a reference signal which slowly changing over time the percentage errors are close to each other. As it can be seen from figure, at the start of the mobile platform movement, the direction angle error of both controllers is higher than speed error. This causes by slippage between the wheel and the ground floor. If we disregard the error occurred at the starting point of sine wave, the maximum percentage errors of sine wave reference for FOPI is $1.43 \%$ and for PI $1.17 \%$ respectively. 

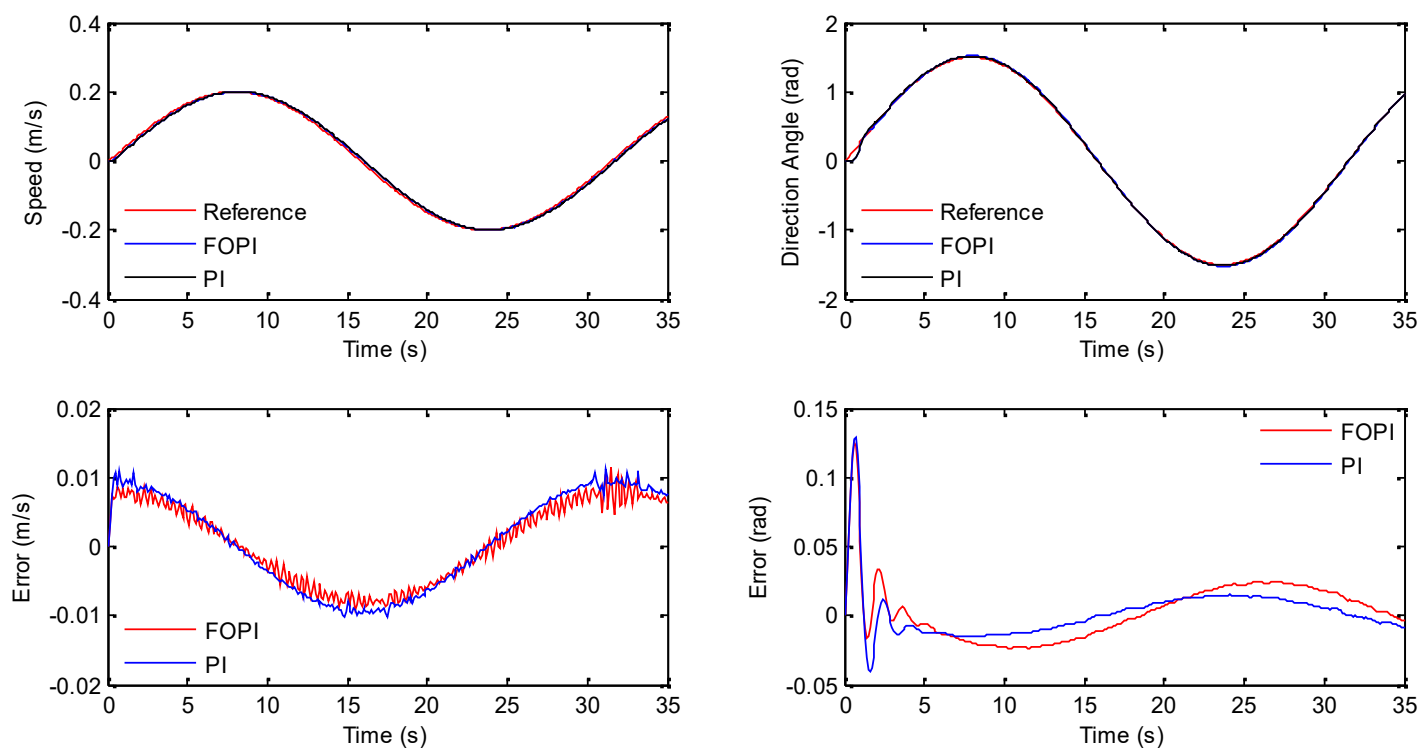

Fig. 5. Experimental results for the sinusoidal speed (a) and sinusoidal direction angle (b) references.
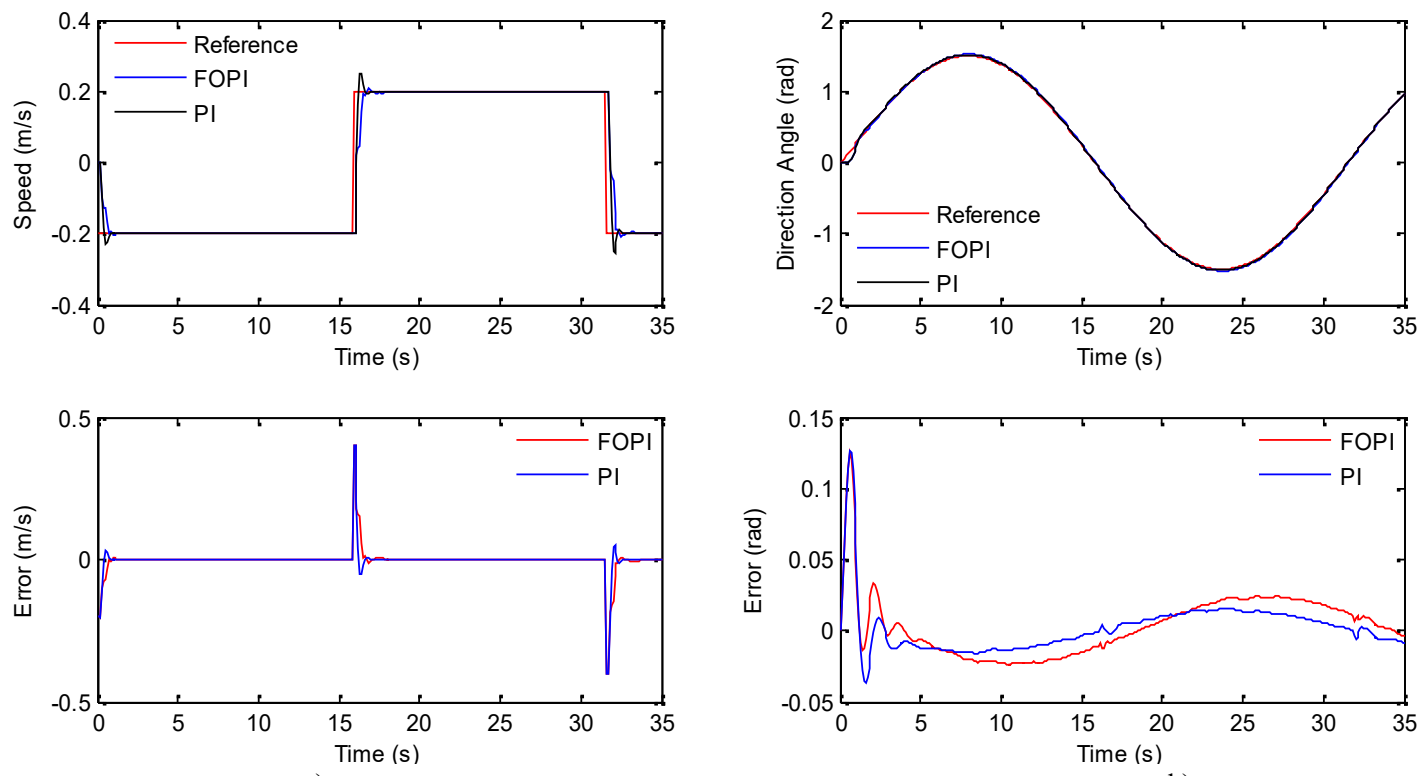

a)

b)

Fig. 6. Experimental results for the square wave speed (a) and sinusoidal direction angle (b) references.
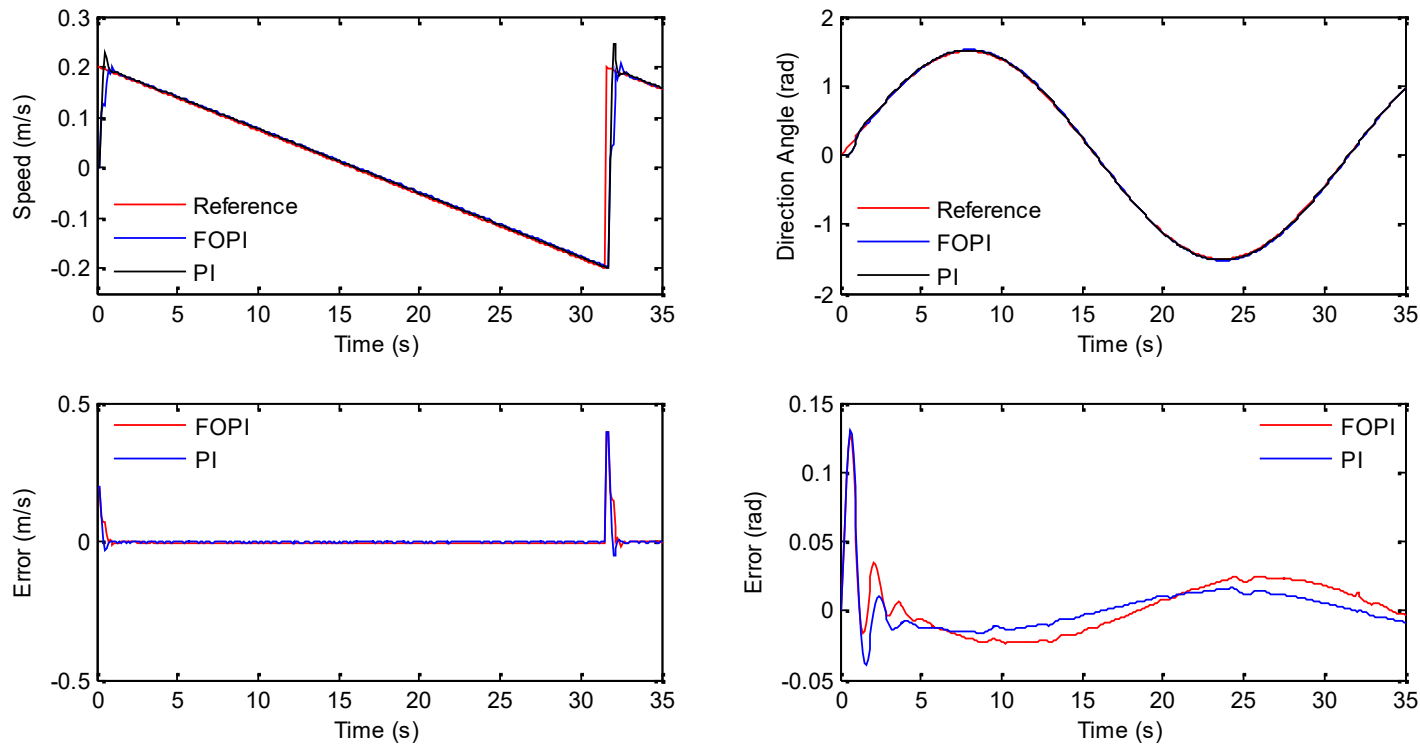

b)

Fig. 7. Experimental results for the sawtooth speed (a) and sinusoidal direction angle (b) references. 
In the second experiment, speed reference is a square wave and direction angle is chosen as sine function for control of $4 \mathrm{WD}$ SSMR. The square wave reference is important to test the performance of the controllers for step changes. It can be seen from Fig. 6 that, when the square wave reference changes, PI gives $0.16 \mathrm{sec}$ rise time with having $24.53 \%$ overshoot while FOPI gives $0.29 \mathrm{sec}$ rise time with having $4.8 \%$ overshoot which is obviously much better. Additionally, when the speed is fixed at $2 \mathrm{~m} / \mathrm{s}$ both controllers displays same steady state performance. On the other hand, if we disregard the error occurred at the starting point of sine wave, the maximum percentage errors of sine wave reference for FOPI is $1.51 \%$ and for PI $1.26 \%$ respectively.

Finally, a sawtooth wave speed and sinusoidal direction angle references are chosen. In Fig. 7, it is observed that, when the sawtooth wave reference sudden changes, PI gives $0.23 \mathrm{sec}$ rise time with having $14.44 \%$ overshoot while FOPI gives $0.41 \mathrm{sec}$ rise time with having $3.84 \%$ overshoot which is smaller than PI. In a similar way, the maximum percentage of errors of the sinusoidal direction angle reference for FOPI is $1.47 \%$ and for PI $1.21 \%$ respectively.

\section{CONCLUSIONS}

In this paper, an experimental study on the application of FOPI controller to a four-wheel skid-steering mobile platform under the different references was presented. In order to show the effectiveness of the FOPI a well-tuned conventional PI controller is also applied. The experimental results show that the FOPI controller shows better steady state performance with having less overshoot and smaller speed error when it compared to the responses of PI. On the other hand, PI gives fast rise time than FOPI and direction angle errors of both controller is similar because of using same reference and slippage between the wheel and the ground floor. To conclude, the applied FOPI controller results in better responses than PI controller to control the speed and direction angle of the vehicle under changing references.

\section{REFERENCES}

[1] J. E. Normey-Rico, et al., "Mobile robot path tracking using a robust PID controller", Control Engineering Practice, vol. 9, no. 11, pp. 1209-1214, 2001. [Online]. Available: http://dx.doi.org/10.1016/ S0967-0661(01)00066-1

[2] P. Zhao, J. J Chen, Y. Song, X. Tao, T. J. Xu, T. Mei, "Design of a control system for an autonomous vehicle based on Adaptive-PID", Int. J. Adv. Robot. Syst, vol. 9, 2012.

[3] T. Das, I. N. Kar, "Design and implementation of an adaptive fuzzy logic-based controller for wheeled mobile robots", IEEE Trans. Control Systems Technology, vol. 14, no. 3, pp. 501-510, 2006. [Online]. Available: http://dx.doi.org/10.1109/TCST.2006.872536

[4] Jiangshuai Huang, et al., "Adaptive output feedback tracking control of a nonholonomic mobile robot", Automatica, vol. 50, no. 3, pp. 821-831, 2014. [Online]. Available: http://dx.doi.org/10.1016/ j.automatica.2013.12.036

[5] Jinzhu Peng, Jie Yu, Jie Wang, "Robust adaptive tracking control for nonholonomic mobile manipulator with uncertainties", ISA Trans., vol. 53, no. 4, pp. 1035-1043, 2014. [Online]. Available: http://dx.doi.org/10.1016/j.isatra.2014.05.012

[6] K. B. Oldham, J. Spanier, The Fractional Calculus. Academic Press, 1974.

[7] I. Podlubny, Fractional Differential Equations. Academic Press, San Diego, California, 1999.

[8] I. Podlubny, "Fractional-order systems and $\mathrm{PI}^{\lambda} \mathrm{D}^{\mu}$ controllers", IEEE Trans. Automatic Control, vol. 44, no. 1, 1999, pp. 208-214. [Online]. Available: http://dx.doi.org/10.1109/9.739144

[9] S. Das, Functional fractional calculus for system identification and controls. Springer, 2008.

[10] D. Valerio, J. Sa da Costa, "Time domain implementation of fractional order controllers", IEE Proc. Control Theory and Appls., vo1. 52, no. 5, pp. 539-552, 2005. [Online]. Available: http://dx.doi.org/10.1049/ip-cta:20045063

[11] D. Xue, Zhao Chunna, Y. Q. Chen, "Fractional order PID control of a DC-Motor with elatic shaft: A case study", in Proc. of American Control Conf., Minnesota, USA, 2006, pp. 3182-3187.

[12] Ying Luo, Chunyang Wang, Yangquan Chen, "Analytical design of fractional order proportional integral and [proportional integral] controllers for robust velocity servo", in Proc. 4th IEEE Conf. Industry Electronics and Applications, Xi'an, China, 2009, pp. 2527.

[13] C. A. Monje, Y. Chen, B. M. Vinagre, D. Xue, V. Feliu, Fractionalorder systems and controls fundamentals and applications. New York: Springer, 2010. [Online]. Available: http://dx.doi.org/10.1007/ 978-1-84996-335-0

[14] H. Ramezanian, S. Balochian, "Optimal design a fractionalorder PID controller using particle swarm optimization algorithm", Int. Journal of Control and Automation, vol. 6, no. 4, pp. 55-68, 2013.

[15] C.-H. Lee, F.-K. Chang, "Fractional-order PID controller optimization via improved electromagnetism-like algorithm", Expert Systems with Applications, vol. 37, no. 12, pp. 8871-8878, 2010. [Online] Available: http://dx.doi.org/10.1016/j.eswa.2010.06.009

[16] J. Cao, J. I. N. Liang, B. Cao, "Optimization of fractional order PID controllers based on genetic algorithms", in the Fourth Int. Conf. Machine Learning and Cybernetics, 2005, pp. 5686-5689.

[17] J. Cao, B. Cao, "Design of fractional order controllers based on particle swarm optimization", in 1st IEEE Conf. Industrial Electronics and Applications, 2006, pp. 1-6. [Online]. Available: http://dx.doi.org/10.1109/iciea.2006.257091

[18] C. A. Monje, B. M. Vinagre, Y. Q. Chen ve V. Feliu, "Proposals for fractional $\mathrm{PI}^{\lambda} \quad \mathrm{D}^{\mu}$ tuning", Fractional Differentiation and Applications, France, 2004.

[19] C. A. Monje, B. M. Vinagre, V. Feliu ve Y.Q. Chen, "Tuning and autotuning of fractional order controllers for industry applications", Control Engineering Practice, pp. 798-812, 2008. [Online]. Available: http://dx.doi.org/10.1016/j.conengprac.2007.08.006

[20] Ying Luo, Yang Quan Chen, "Fractional-order [proportional derivative] controller for robust motion control: Tuning procedure and validation", in Proc. of the American Control Conf. St. Louis, Missouri, 2009.

[21] A. Al-Mayyahi, W. Wang, P. Birch, "Design of fractional-order controller for trajectory tracking control of a non-holonomic autonomous ground vehicle", Journal of Control, Automation and Electrical Systems, pp. 1-14, 2015.

[22] B. Abdullah, A. Derdiyok, "Real-time velocity and direction angle control of an automated guided vehicle", Int. Journal of Robotics and Automation, vol. 29, no. 3, 2014

[23] B. Abdullah, A. Derdiyok, "Sensorless velocity and direction angle control of an unmanned vehicle", Turkish Journal of Electrical Engineering \& Computer Sciences, vol. 2, no. 4, pp. 580-589

[24] P. Jagatheeswari, J. Renisha, "Modellingof BLDC motor for robotic leg joint", Journal of Advances in Mechanical Engineering and Science, vol. 1, no. 1, pp. 11-21, 2015. [Online]. Available: http://dx.doi.org/10.18831/djeee.org/2015011002

[25] O. J. Oguntoyinbo, "PID control of brushless DC motor and robot trajectory planning simulation with MATLAB ${ }^{\circledR} /$ SIMULINK $\AA$ ", 2009 . 